\title{
Quartz Channel Fabrication for Electrokinetically Driven Separations
}

\author{
C. M. Matzke, D. W. Arnold, C. I. H. Ashby, S. H. Kravitz, M.
Sandia National Laboratory
Albuquerque New Mexico, 87185
}

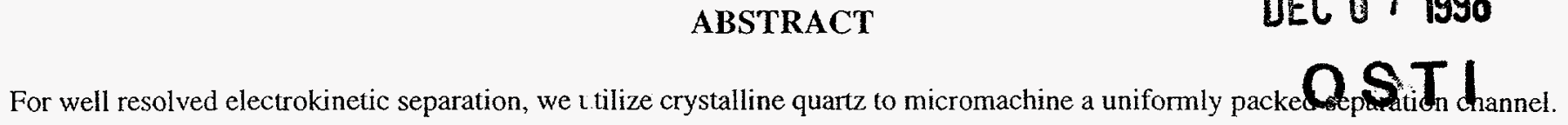

DEC 071998 Packing features are posts $5 \mu \mathrm{m}$ on a side with $3 \mu \mathrm{m}$ spacing and etched $42 \mu \mathrm{m}$ deep. In addition to anisotropic wet etch characteristics for micromachining, quartz propirties are compatible with chemical solutions, electrokinetic high voltage operation, and stationary phase film depositions. To seal these channels, we employ a room temperature silicon-oxynitride deposition to form a membrane, that is subsequently coated for mechanical stability. Using this technique, particulate issues and global warp, that make large area wafer bon ting methods difficult, are avoided, and a room temperature process, in contrast to high temperature bonding techniques, accommodate preprocessing of metal films for electrical interconnect. After sealing channels, a number of macro-assembly steps are required to attach a micro-optical detection system and fluid interconnects.
\end{abstract}

RECEIVED

Keywords: microchannel, integrated channel, micromachined channel, packed channel, electrokinetic channel, electrophoretic channel

\section{Introduction}

Electrokinetically driven separation methods de' reloped to analyze complex chemical mixtures have been implemented in a number of biochemical, environmental, and industrial applications. Typically, these separation technologies employ capillary tubing with bore sizes from $<5$ to $>500$ microns. While the capillary separations are quite powerful, recent efforts have demonstrated that fabrication of planar separaticn systems using microelectronics fabrication techniques provide key advantages over the capillary-based approach. These advantages in the areas of sample manipulation, heat dissipation, robustness, etc., allow separations under conditions that are not sasily accessible in capillary-based experiments. In the planar front, it is possible to integrate detection and electronic systems with the same methodologies, providing a new class of analytical tools for the researcher. In addition, micro-processing facilitates device design change for separation optimization, and thin film processing, that can be instrumental in stationary phase research.

To fabricate these superior devices, researchers are employing microelectronic patterning technology, advanced deep etching, and novel sealing techniques. Stringent electrokinetic parameter, however, complicate the fabrication of these devices. For example, typical capillary-based separation will operate with an applied field of $>200 \mathrm{~V} / \mathrm{cm}$ across a $30 \mathrm{~cm}$ length of $50 \mu \mathrm{m}$ diameter capillary. The solution used for separation can have a mixture of aqueous and organic buffers/solvents ranging from $\mathrm{pH} 4$ to $\mathrm{pH} 10$. While the broad knowledge base and attractive integration prospects make silicon a likely candidate for fabricating these structures, its conductivity at the anticipated operating voltages require the use of very high integrity dielectric film to prevent leakage currents through the sulstrate. Glasses, due to electrical insulating properties and compliance with thermal bonding methods for sealing the fluid channels, have been popular for fabricating these systems. Channels also have been successfully fabricated by micromachining and molding plastics. Separations in these devices are currently limited to those that are compatible with the particular chemicals used in the separation solutions. Since the minimum size feature, which can be fabricated in glass or plastic is lir ited, most systems to date have used open channels with a cross-section area on the order of $400 \mu \mathrm{m}^{2}$.

The majority of these systems rely upon capillcry electrophoretic separations, which separate components of the chemical mixture based only upon charge-to-mass ratio. To separate uncharged compounds, methods like capillary electrochromatrography differentiate between compounds based upon their chemical properties. By flowing the mixture in solution over a coated surface, a partitioning between the running solution and surface coating molecules, causes separation of the mixture into its individual components. Fo: either capillary-based separation, performance is enhanced by increasing the 


\section{DISCLAIMER}

This report was prepared as an account of work sponsored by an agency of the United States Government. Neither the United States Giovernment nor any agency thereof, nor any of their employıes, make any warranty, express or implied, or assumes any legal liability or responsibility for the accuracy, completeness, or usefulness of any information, apparatus, procluct, or process disclosed, or represents that its use would not infringe privately owned rights. Reference herein to any specific commercial product, process, or service by triade name, trademark, manufacturer, or otherwise does not necessarily constitute or imply its endorsement, lecommendation, or favoring by the United States Governrnent or any agency thereof. The views and opinions of authors expressed herein do not necessarily state or reflect those of the United States Government or any agency thereof. 


\section{DISCLAIMER}

Portions of this document may be illegible in electronic image products. Images are produced from the best available original document. 
surface area, and to accomplish this requirement, the stationary phase is put onto a support ( $\mu \mathrm{m}$-sized silica spheres) which are packed into the capillary. In planar micro-channel format, one would like to be able to fabricate the micro-support into the channel then apply coatings of choice.

A promising material for accomplishing this goćl is crystalline quartz. Due to its crystalline properties, this electricallyinsulative material has a characteristic anisotropic wet etching behavior appropriate to micromachining. Being a special form of silicon dioxide the same well-developed stationary phase coating technologies developed for capillary-based separations can be directly applied to our device. We start our discussion of this device with the separation techniques to be employed and the advantages of electrokinetically-driven chip-based systems over capillary-based separations. We follow with details of device fabrication with emphasis on quartz etching and development of our membrane sealing process.

\section{Separation Design Considerations}

In the liquid phase, a variety of criteria can be selected based on solution and channel electrochemistries such as mass-to-charge ratio, size, hydrophobicity, etc. to separate a mivture into its components. A number of methods have been developed for such separations using electrokinetically-driven methods in capillaries: electrophoresis, electrochromatography, micellar electrochromatography, affinity chromatography, and gradient chromatography. Each method allows one to separate mixtures based upon a different characteristic. Appropriate combinations of methods provide orthogonal information about the mixture composition.

For electrokinetically-driven separations, an electric potential is applied between two buffer reservoirs connected by a small cross-sectional area tube or channel. Interaction of the channel's inherent surface charges with the buffer solution produces a charge 'double-layer'. The applied voltage induces a motion of the mobile solution ions, relative to the surface, carrying the buffer along the length of the channel with a very flat plug-flow profile. This phenomenon, known as electro-osmotic flow, allows the transport of fluid through narrow channels without the dispersion induced by the parabolic-flow associated with the alternative pressure-driven flow. Additionally, flectro-osmotic flow can be used to transport the same fluid through packed beds that would be beyond the normal capabilities of pressure-based systems because the particles provide a driving force rather than a resistive force.

An integral part of the separation process is sample injection, detection, and electronic configurations. For separation of a mixture, only a small volume $(\leq 1 \mathrm{~nL})$ plug of sample needs to be injected onto the column. An important advantage of planar channels over capillary tubes, lies in the ability to manipulate fluid through a micromachined manifold by switching voltages. In this way, uniform sample injections onto the separation column can be repeated with ease. The volume of injection can be controlled by either the geometric design of the manifold or by a voltage pulsing sequence, both of which allow $\mathrm{pL}$ control of sample injection. Another significant advantage in the planar approach is the ability to design parallel separation channels lithographically, which is a much more difficult task to accomplish in a capillary-based format. Planar systems also allow one to design and employ in tegrated detection systems and electronics, which is not as plausible for capillary systems.

The type of separation to be accomplished depends upon the preparation of the channel prior to sample injection. In the area of micro-processed devices, effort has been primarily been focused on electrophoretic separations of species based upon their massto-charge ratio. This limits the system's separation capabilities to a small subset of the compounds one would like to separate in rapid, efficient manner. For separation of the remaining large classes of uncharged compounds, electrochromatographic methods must be employed. The most commonly used method is reverse-phase capillary electrochromatography (CEC). In this method, the mixture separates based upon he partitioning of the compounds between the buffer and a coating on a support material in the channels. By varying the characteristics of these coatings and the buffer, the selectivity and performance of the column can be tuned to meet then needs of the separation.

For the development of planar separation systems, it is desirable to fabricate the micro-support structures in the system. In this way, one can imagine designing arrays of features that can improve separation performance by eliminating sources of dispersion associated with beds of packed spheres. To assess the feasibility of microfabricating successful planar chromatographic separation systems, it is useful to consider the properties of supports used in current capillary systems. Typically. 
chromatographic supports are small diameter $(1.5-3 \mu \mathrm{m})$ silica spheres. As the sphere diameter decreases, the surface area available for partitioning to occur along the leng th of the column increases. Silane chemistry is used to coat the sphere uniformly, typically with a long-chain hydrocarbon. For exceptional separation performance, it is very important that the spheres have a very narrow size distribution. Ot nerwise, eddy diffusion leads to a dispersion of the sample as it propagates through the system.

Lithographic methods can produce features on the size scale required for separation quite easily. Additionally, the size distribution is excellent due to the achievable lithographic resolution. The primary limitation is the identification of a fabrication material that can be etched with such small features yet still operate at the high voltages required for electrokinetic separations. As mentioned in the Introduction, current favored methods of fabrication in glass or plastics are not amenable to such designs. Silicon, while excellent for small feature fabrication, has the complication of being conductive. We find that the use of crystalline quartz as the fabrication mater:al allows an acceptable compromise between the two materials. We are able to produce small features quite easily in this material as will be described below. The material is an inherent insulator and the silane coating chemistry is directly applicable since the material is silicon dioxide. The material has the added advantage that it is optically transparent over a wide range of wavelengths, which is important when considering the integration of optical detection systems.

\section{Device Fabrication}

The present device configuration requires two-si Jed micro and macro-processing of the quartz. Initial fabrication steps involve configuring either side of the quartz slice to form the packed channel for separations and the open channels for sample injection as detailed in our quartz etching section. The next integrated fabrication step is deposition of electrodes at the channel ends. To define electrodes, we utilize a shadow mask on sither side of the quartz slice and a rotating stage for metalization rather than photolithography over our deep etched topographies. The final integrated process is deposition of the room temperature membrane seal on the packed channel described in the membrane seal fabrication section.

The remaining fabrication steps to complete the device are macro processing steps described next. First, micro-fabricated fluorescence detection optics ${ }^{1}$ are aligned through the clear membrane to the channel near the end as depicted in Fig. 1 and attached to the membrane using UV curing acrylic adhesive, which is a close index match to the two silica surfaces. Secondly, the remainder of the membrane is coated with a mechanically robust film to protect the membrane during device handling. We

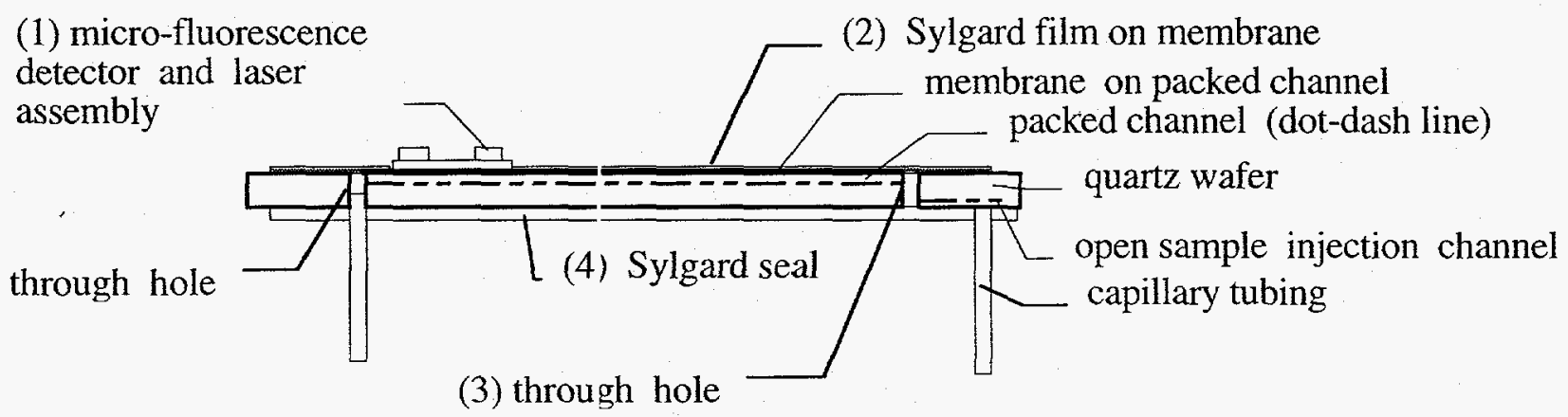

Figure 1. Schematic of micro-channel device for electrokinetic separation.

use Sylgard, a two part elastomer material that forms a tough clear coating after a room temperature cure, for applications requiring a material for device stability. Third, to access the packed channel, holes are ground from the bottom sample injection side through the quartz to the Sylgard coating at either end of the packed channel as depicted in Fig. 1. Fourth, to seal the open sample injection channels, we utilize a Sylgard technique ${ }^{2}$ we have demonstrated for sealing channels. Finally, for solution injection and removal, capillary tubing is attached using epoxy at the sample injection access ports in the Sygard seal assembly. 


\subsection{Quartz etching}

Crystalline quartz has a fast etching Z-direction, that is never shown as a facet in familiar naturally grown crystals as shown in Fig. 2 The Z-direction is instead composed of a rather complex set of planes labeled as $R, r, s$, and $x$. The $x$ and $y$ directions, on the other hand are composed of only the slow etching $m$-planes. Commercially prepared quartz wafers oriented as Z-slices, are cut across the ingot perpendicular to the Z-direction and in etching a Z-slice, one or more of the Rrsx-facets appear.
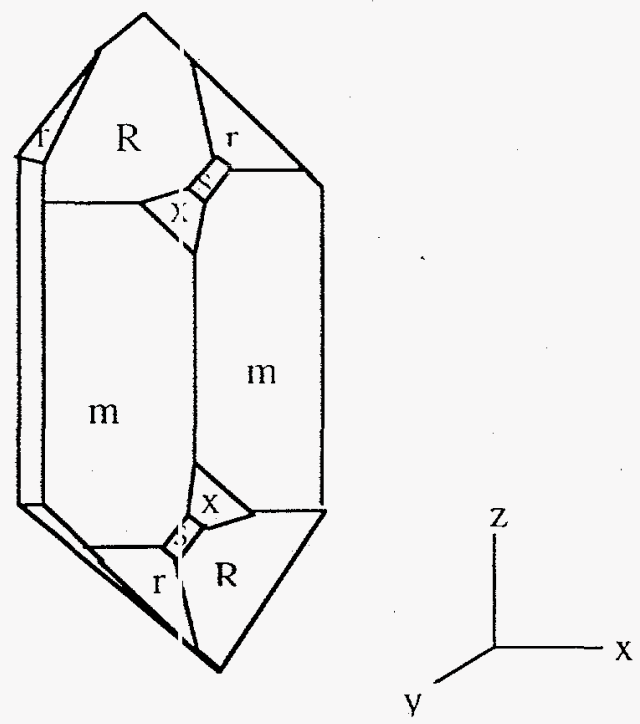

Figure :2 Schematic of crystalline quartz

A number of studies and models of facet etching rate dependence on solution concentration, stirring, temperature, and orientation have been reported, but theoretical etching conclusions for crystalline quartz are still in contention. ${ }^{3.456 .7}$ From our etch results, ion diffusion limitations in solution were quite apparent. We patterned Z-cut quartz using a test mask that

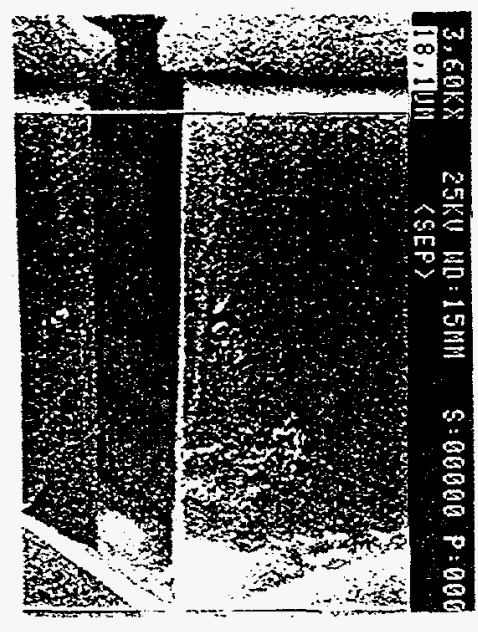

3.a

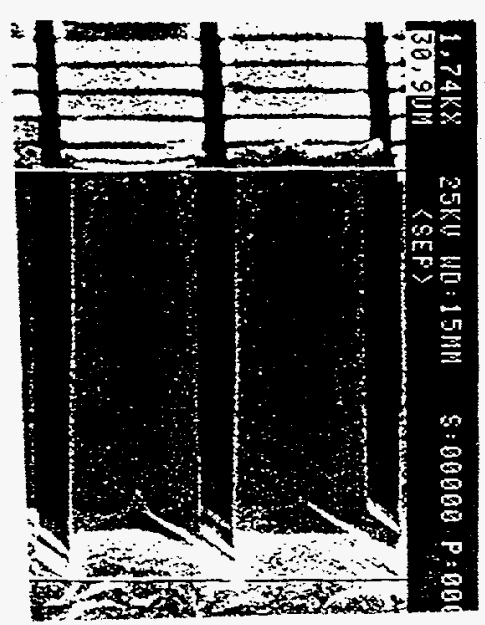

3.b

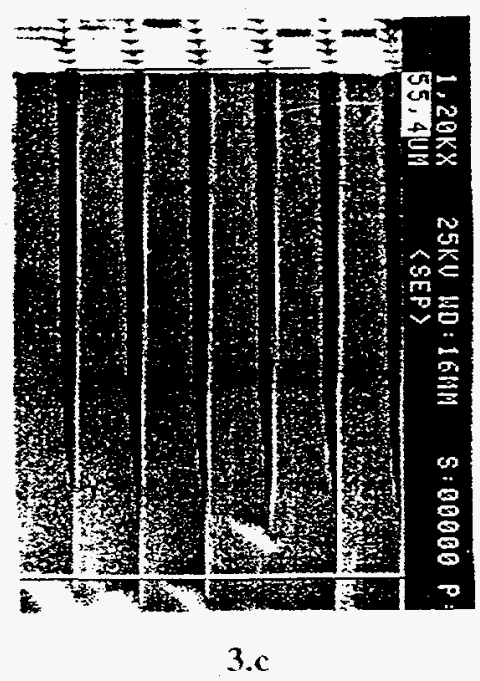

3.c

Figure 3 From left to right etch depths and box sizes: a. $18 \mu \mathrm{m}$ deep for $20 \mu \mathrm{m}$ box, b. $30 \mu \mathrm{m}$ dieep for $10 \mu \mathrm{m}$ box, and c. $55 \mu \mathrm{m}$ deep for $5 \mu \mathrm{m}$ box. 
contained arrays of 30 boxes on a side and box sizes ranging from 4 to $20 \mu \mathrm{m}$ with $3 \mu \mathrm{m}$ sized gaps between boxes. All arrays were configured on the same Z-cul slice, so edge orientations were identical. As shown in Fig. 3, shallower etch depths consistently resulted for larger box anrays compared to small box arrays with the same spacing. Our results appear to be a diffusion limitation in reverse, in other words, ecch rates increase as the solution becomes more dilute by etch reactants ${ }^{x}$. Based on these preliminary results. we designed our separation channel with $5 \mu \mathrm{m}$ square packing features to maximize etch depth.

To fabricate the quartz wafer for the electrophorctic device, (wo-sided wet processing of quartz is required to configure the packed channel on one side and open crossed channels tor sample injection on the second side. For this processing, Z-cut quartz starting material obtained from Boston Piczo - Optics Inc. was masked with patterned $\mathrm{Cr} / \mathrm{Au}$ heforc the $\mathrm{HF}$ etch. $\mathrm{The} \mathrm{Cr} / \mathrm{Au}$ was patterned on each side using photolithograp hy followed by dry etching the Cr/Au with argon milling on the packed channel side and wet clching the $\mathrm{Cr} / \mathrm{Au}$ in chromium etchant and $\mathrm{KI} / \mathrm{I}_{2}$ on the open channel side. For the double-sided processing, one side was protected with mylar sheeting or photoresist while the opposite side was processed. In etching the micro-structures, wo soaked the patterned slice in $49 \%$ HF with no st rring due to uninvestigated diffusion effects and uniformity concerns. The etch depth of the packed channel secms to siturate a. $42 \mu \mathrm{m}$ in $8 \mathrm{hr}$. for our present $5 \mathrm{~cm}$. Fong serpentine shaped channel that is 80 $\mu \mathrm{m}$ wide with the 5um packing features. As stown in the channel portion of Fig. 4, other than some comer etching, mask undercutting was minimal due to well finished chem-mechanically polished starting surfaces. The last step in the channel etch process was removal of the Cr/Au mask.

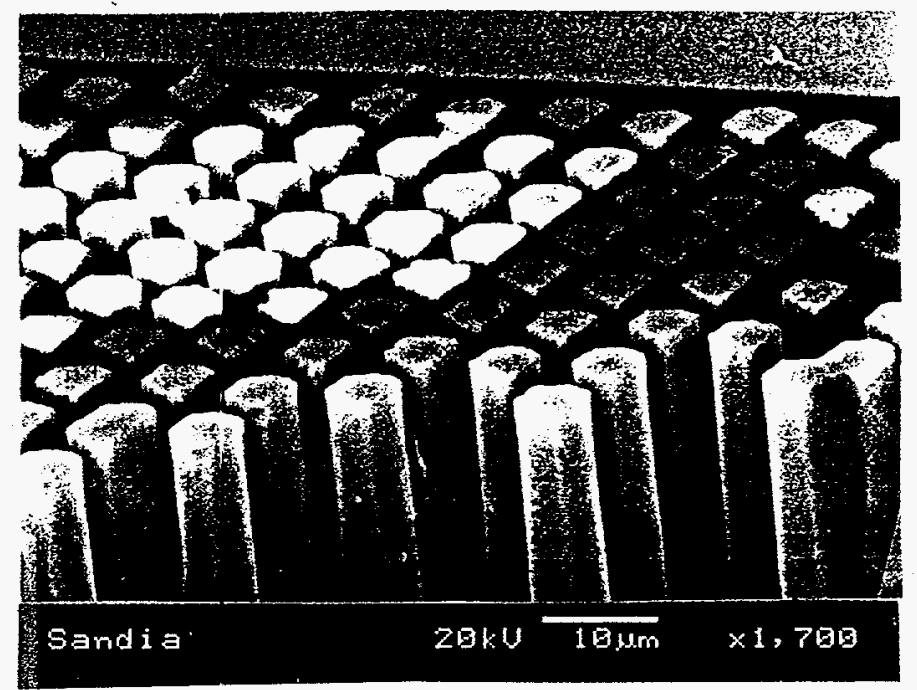

Figure 4 Cut away portion of packed channel after etching, indicates array uniformity except at details of post configurations.

\subsection{Membrane Seal Fabrication}

To form a membrane seal over etched packed channels, we utilize a sacrificial photoresist and multiple depositions of a newly developed room temperature silicon-oxynitride $\left(\mathrm{Si}_{1} \mathrm{O}_{3} \mathrm{~N}\right.$, ) film (referred to as oxynitride). "In first processing steps as depicted in Fig 5. photoresist is patterned to fill the etche I channel arcas except at the channd sides, where subsequent windows are required to allow dissolution of resist. For this procedure we used positive tone OCG thick photoresist, a short spin time to minimize resist flow out of the etched channel, and a doubled exposure time $(8 \mathrm{sec}$ ) for the thicker resist that results with channel fill. After developing exposed resist, an over-develop time of $1 \mathrm{~min}$, followed by an oxyen plasma descum wals utilized 10 clear resist from tops of post structures. As shown in Fig. 5, after resist processing. resist is between posts, cleared from tops of posts. and partially cleared from the channel sides. 


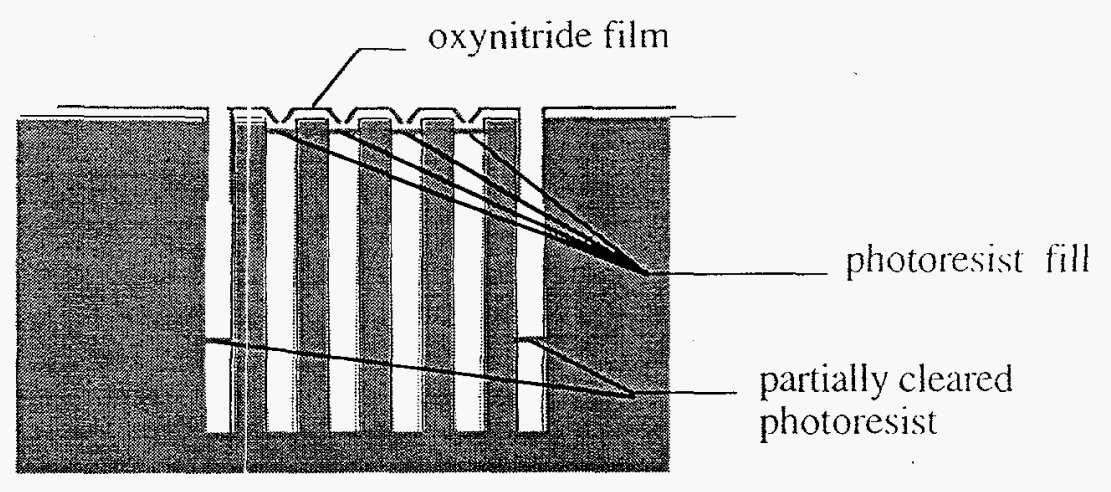

Figure 5 Schematic of photoresist processing and first oxynitride film

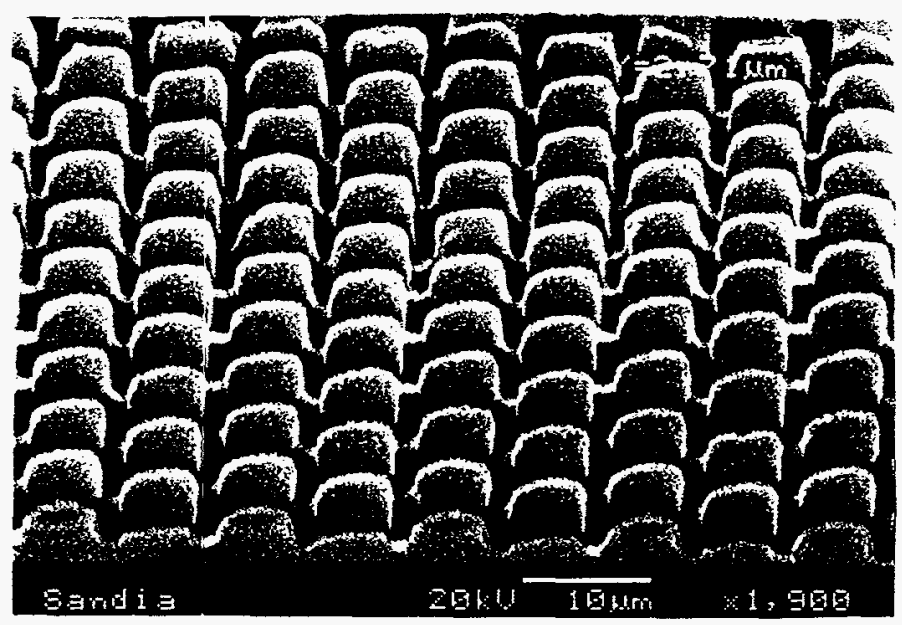

$6 . \mathrm{b}$

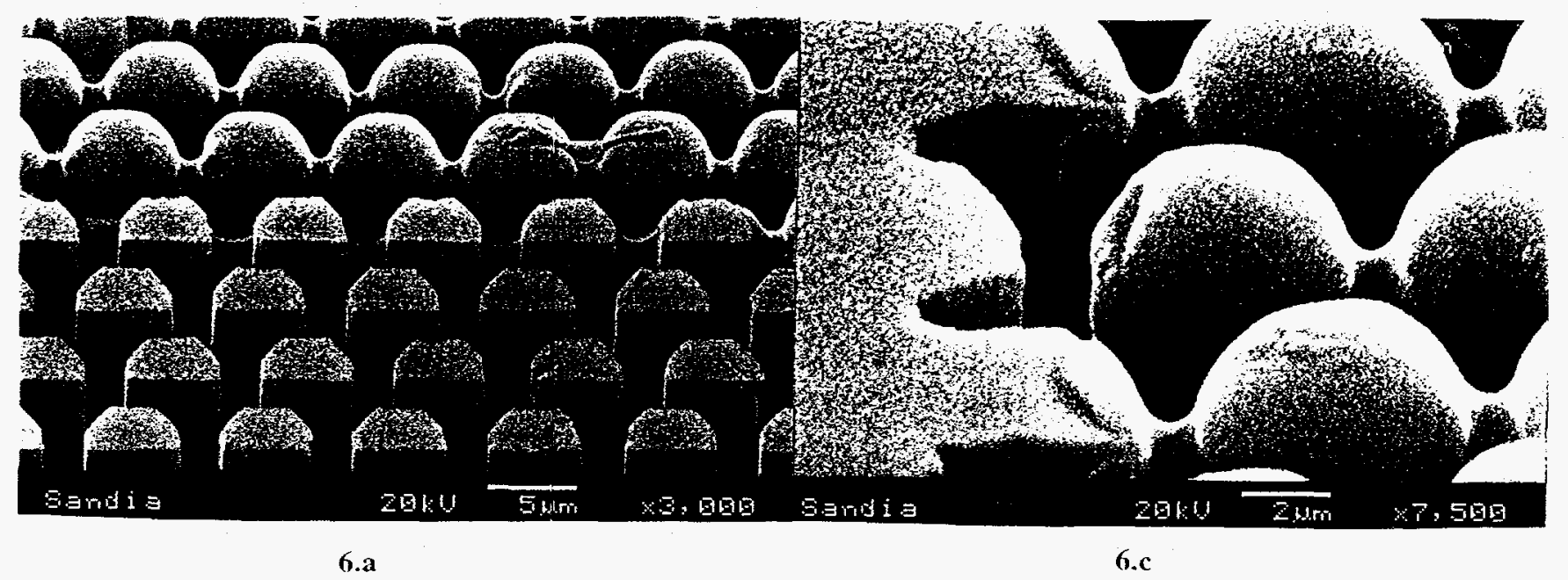

Figure 6 SEM views of channel areas after the first oxynitride deposition.

The next process is the tirst room temperature oxynitride deposition. A 30 min. deposition resulted in a film thickness of $3500 \AA$ A. From Fig. 5, film coverage occurs over the center area of the channel, and not on the channel sides that do not have photoresist fill. Afrer the film deposinom. pho.oresist was removed via the windows at the channels sides by soaking the guaty 
wafer in acetone for several ( 3 to 5) hours. At this point some samples were examined with scanning electron microscopy (SEM). From Fig. 6a that shows a channel portion with the membrane partially removed, we found the membrane adequately attached to the post configurations and configurations were cleared of resist. From Fig. 6b, the oxynitride film is conformal over the channel width except as shown in Fig. 6c, where the window area at the channel side is shown. This result is consistent with the configuration depicted in Fig. 5, that shows no oxynitride film at channel sides that did not have resist fill. The second room temperature oxynitride film of $1.2 \mu \mathrm{m}$ was deposited at a rate 5 times faster than that of the first. From Fig. $7 \mathrm{a}$ taken at the same area as Fig. 6c, the second teposition appears to adequately close the previous window gap, however at the other channel edge shown in Fig. 7b, an openin: still appears at the larger square feature and a third deposition was required to complete film coverage over the channel.

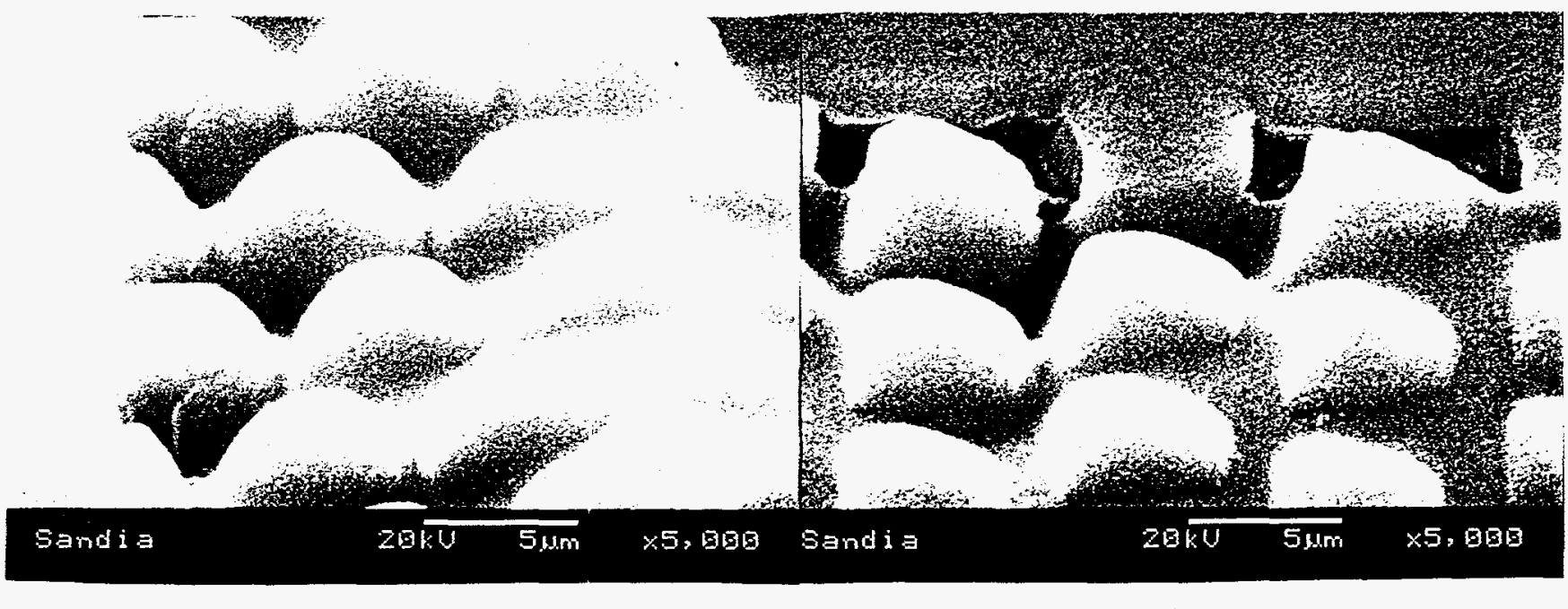

7.a

7.b

Figure 7 SEM views of channel regions after second oxynitride deposition

From processing results, oxynitride membrane soverage for this application depends primarily on the initial etch configuration of the channel and clearing photoresist from tops of post structures. From SEM inspections, the window size at the channel side after the first deposition was determined by the etched gap between the channel side and the first post next to the side in the window areas. Therefore, the etch mask must contain etch gaps at the channel sides adequately large for resist dissolution, but not so wide that excessive oxynitride is require 1 for gap coverage. We also noted that film adherence to the tops of the post structures, that were previously cleared of resist, was important for successful coverage. Using this method to seal channels, yield is primary controlled by process design rather than the area and particulate dependencies of wafer bonding techniques.

\section{Summary}

For electrokinetic separations, channel boundary layer interaction with sample solutions and channel feature uniformity are key to optimum device performance. Using crystalline quartz, which is compatible with rather stringent electrokinetic separation requirements, we etched the required small unilorm micron-sized features to form a packed channel $5 \mathrm{~cm}$ long in a planar format. To seal these channels, we utilize a newly developed room temperature oxynitride film ${ }^{12}$ to form a membrane that is subsequently coated for mechanical stability. With room temperature deposition, the resist does not bake as with conventional CVD films, and resist is easily removed. Depcisitions are relatively fast at 100 's $\AA / \mathrm{min}$. for required thicknesses of a few microns. And film compositions are compatit le to stationary phase chemistry. Using this channel sealing technique, high fusion bonding temperatures in the $1200 \mathrm{C}$ range for quartz and particulate problems associated with sealing large areas are both avoided. To complete fabrication a number of nacro-processing steps are necessary to assemble a micro-fluorescence detector and complete the sample injection system 


\section{Acknowledgments}

Sandia is a multiprogram laboratory operated by Sandia Corporation, a Lockheed Martin Company, for the United States Department of Energy under Contract DE-AC04.94AL85000

\section{References}

1 M.E. Warren, R.F. Carson, W.C. Sweatt, J.R. Wendt, J.A. Nevers, M.H. Crawford and H.Q. Hou, "VCSEL Applications in Sensors and Microsystems, "Photonics West 98, Jan 25, 1998, San Jose, CA (published in SPIE Proc.3286, 42-51, (1998)).

2 We use Sylgard 184 to seal open channels. (ilass cover slips are first cleaned by soaking in a 1:1:5 mixture of NH4OH:H2O2:H2O. The 10:ISylgard is mixed and then degassed in a vacuum until bubbles no longer appear. Next the Sylgard is dispensed on the glass cover slip and spun $45 \mathrm{~s} @ 500 \mathrm{rpm}$ to distribute the Sylgard, and then $45 \mathrm{~s} @ 2000 \mathrm{rpm}$ to form a thin layer. In the final step the prepared glass cover slip with film is contacted to the wafer with etched channels and either cured at $90 \mathrm{C}$ for a few hours or at room temperature cured over 24 hours. Longer cures result in the best seals.

3 Pelle Rangsten, Christer Hedlund, Ilia V Kalardjiev, and Ylva Backlund; J. Micromech, Microeng, 8 (1998) I-6

4 J. K. Vondeling, J. Mater. Sci.; 18 (1983) 304-314

5 C. R. Tellier, T. G. Leblois; IEEE International Frequency Control Symposium; (1993)

6 J. S. Danel, F. Michel, G. Delapierre; Sensors and Actuators, A21-A23 (1990) 971-977

7 Christer Hedlund, Ulf Lindberg, Urban Bucht, Jan Soderkvist; Micromech, Microeng, 3 (1993) 65-73

8 C. M. Matzke and D. L. Kendall manuscript in preparation .

9 C.I.H. Ashby and M.M. Bridges, manuscript in preparation. 